\title{
Quantization of a 3D Nonstationary Harmonic plus an Inverse Harmonic Potential System
}

\author{
Salim Medjber, ${ }^{1}$ Hacene Bekkar, ${ }^{2}$ Salah Menouar, ${ }^{3}$ and Jeong Ryeol Choi ${ }^{4}$ \\ ${ }^{1}$ Department of Material Sciences, Faculty of Science, University of M'sila, 28000 M'sila, Algeria \\ ${ }^{2}$ Faculty of Technology, University of Ferhat Abbas Sétif-1, 19000 Sétif, Algeria \\ ${ }^{3}$ Laboratory of Optoelectronics and Compounds (LOC), Department of Physics, Faculty of Science, \\ University of Ferhat Abbas Sétif-1, 19000 Sétif, Algeria \\ ${ }^{4}$ Department of Radiologic Technology, Daegu Health College, Daegu 41453, Republic of Korea \\ Correspondence should be addressed to Jeong Ryeol Choi; choiardor@hanmail.net
}

Received 27 October 2015; Accepted 28 March 2016

Academic Editor: Yao-Zhong Zhang

Copyright (C) 2016 Salim Medjber et al. This is an open access article distributed under the Creative Commons Attribution License, which permits unrestricted use, distribution, and reproduction in any medium, provided the original work is properly cited.

\begin{abstract}
The Schrödinger solutions for a three-dimensional central potential system whose Hamiltonian is composed of a time-dependent harmonic plus an inverse harmonic potential are investigated. Because of the time-dependence of parameters, we cannot solve the Schrödinger solutions relying only on the conventional method of separation of variables. To overcome this difficulty, special mathematical methods, which are the invariant operator method, the unitary transformation method, and the Nikiforov-Uvarov method, are used when we derive solutions of the Schrödinger equation for the system. In particular, the Nikiforov-Uvarov method with an appropriate coordinate transformation enabled us to reduce the eigenvalue equation of the invariant operator, which is a second-order differential equation, to a hypergeometric-type equation that is convenient to treat. Through this procedure, we derived exact Schrödinger solutions (wave functions) of the system. It is confirmed that the wave functions are represented in terms of time-dependent radial functions, spherical harmonics, and general time-varying global phases. Such wave functions are useful for studying various quantum properties of the system. As an example, the uncertainty relations for position and momentum are derived by taking advantage of the wave functions.
\end{abstract}

\section{Introduction}

The quantum dynamics of central potential systems that is originally developed in the context of stationary Hamiltonian systems [1-3] can be extended to more complicated systems that involve time-dependent parameters. Time-dependent Hamiltonians are useful for describing nonstationary quantum dynamical systems which are ubiquitous in the physics world. Among many time-dependent Hamiltonian systems, central potential systems with time-dependent parameters have attracted considerable interest in the literature because they provide solvable models for various physical systems. As a consequence, active researches have been carried out in this context, accompanying lots of reports so far [4-16]. For instance, a dissipative harmonic oscillator perturbed by an inverse quadratic potential described with time-dependent parameters has been studied [7]. Quantum properties of harmonic motion of a charged particle that have timedependent effective mass under time-dependent singular potential have been investigated [16]. However, the topics of most of these researches were limited to the case of one- or two-dimensional systems due to the difficulty in developing mathematical procedures for three- or higher-dimensional systems that involve time-dependent parameters.

For a full understanding of profound physical properties of such systems, quantum analytical solutions of corresponding Schrödinger equations, as well as classical solutions, are necessary. Various mathematical tools and methods for solving Schrödinger equations of complicated time-dependent Hamiltonian systems have been suggested and developed up to now. They are the invariant method [17], the method based on path integral formulation [18], the trial function method [19], the canonical/unitary transformation approach [20], and so on. In particular, the invariant method which 
was devised by Lewis and Riesenfeld [21, 22] is very useful for unfolding fundamental theories of quantum dynamics for the case where the Hamiltonian of the given system explicitly depends on time. Among diverse nonstationary quantum systems, the time-dependent quantum system with a harmonic plus an inverse harmonic potential in one or two dimensions is one of the interesting problems that has attracted permanent interest on account of its emerging applications in actual physical systems [9, 16, 23, 24]. For instance, exact quantum solutions for many body systems $[2,3]$ and polyatomic molecules [23] and the quantum behavior of a charged particle in electromagnetic fields [24] can be investigated through this model. These studies for nonstationary quantum systems may be possibly extended to more complicated systems, such as the time-dependent quantum systems with spherical harmonic plus an inverse harmonic potential in three dimensions.

Stimulated by this trend for recent researches, we investigate exact quantum solutions of the time-dependent harmonic potential system perturbed by an inverse harmonic potential in three dimensions in the present work. To this end, several particular mathematical procedures will be employed in order to overcome the mathematical difficulty for unfolding quantum theory under the consideration of time-dependent parameters in three dimensions. Typically, the Schrödinger solutions or wave functions for a timedependent Hamiltonian system are represented in terms of the eigenfunctions of an invariant operator of the system. Such eigenfunctions are obtained by solving the eigenvalue equation of the invariant operator. Because the invariant operator is represented in terms of some time functions, the direct evaluation of the eigenvalue equation is somewhat difficult. This difficulty can be resolved by introducing the unitary transformation method and the Nikiforov-Uvarov (NU) method together. The eigenvalue equation can be simplified by unitary transformation with an appropriate unitary operator. The NU method enables us to solve such arbitrary-type second-order differential equations.

\section{Hamiltonian and Invariant Operator}

As is well known, the study of quantum dynamical properties of a system requires Schrödinger solutions as a preliminary step. While quantum features of time-dependent harmonic plus an inverse harmonic potential in one or two dimensions are extensively studied in the literature, the same problems in three dimensions are not extensively studied yet as far as we know. As a solvable dynamical system in three dimensions, we consider a time-dependent harmonic plus an inverse harmonic potential with a linearly increasing mass $[25,26]$. In this case, the Hamiltonian has the form

$$
\begin{aligned}
H(\vec{r}, p, t)= & \frac{1}{2 M_{0}(1+\varepsilon t)^{2}} p^{2}+\frac{1}{2} M_{0}(1+\varepsilon t)^{2} \omega_{0}^{2} e^{k t} r^{2} \\
& +\frac{\delta}{2 M_{0}(1+\varepsilon t)^{2}} \frac{1}{r^{2}},
\end{aligned}
$$

where $\omega_{0}, M_{0}, k, \delta$, and $\varepsilon$ are real constants. Notice that the linearly increasing mass appears in several dynamical systems such as the expanding shells with mass accretion in a plasma [25] and the mass-accreting Algol-type eclipsing binaries [26]. Simple classical modeling of a system with a linearly increasing mass has been carried out by Kunkel and Harrington [27]. A pail-rain model of a linearly massaccreting system is described in $[28,29]$. Here, we consider only a slowly time-varying function for convenience, which is viable under the levy of a condition $\varepsilon \ll 1$.

The momentum operator in three dimensions is defined as

$$
p^{2}=p_{r}^{2}+\frac{L^{2}}{r^{2}}
$$

where

$$
p_{r}=-i \hbar\left(\frac{\partial}{\partial r}+\frac{1}{r}\right) \text {, }
$$

and $L$ is the total angular momentum of the form

$$
L^{2}=-\hbar^{2}\left[\frac{1}{\sin ^{2} \theta} \frac{\partial^{2}}{\partial \varphi^{2}}+\frac{1}{\sin \theta} \frac{\partial}{\partial \theta}\left(\sin \theta \frac{\partial}{\partial \theta}\right)\right] .
$$

Notice that the position and the momentum operators satisfy the commutation relation $\left[r, p_{r}\right]=i \hbar$.

To investigate quantum features of the system, it is necessary to solve the Schrödinger equation in the first place. Let us express the Schrödinger equation for this system in the form

$$
\begin{aligned}
& {\left[\frac{1}{2 M_{0}(1+\varepsilon t)^{2}}\left(p^{2}+\frac{\delta}{r^{2}}\right)+\frac{1}{2} M_{0}(1+\varepsilon t)^{2} \omega_{0}^{2} e^{k t} r^{2}\right]} \\
& . \Psi(\vec{r}, t)=i \hbar \frac{\partial}{\partial t} \Psi(\vec{r}, t) .
\end{aligned}
$$

Because this equation is a three-dimensional form that involves time-dependent parameters, it may not be an easy task to solve it. Hence, special mathematical techniques are necessary. To solve this equation, we first use the dynamical invariant method devised for evaluating quantum solutions of time-dependent Hamiltonian systems by Lewis and Riesenfeld [22]. This method can be developed through an establishment of a nontrivial Hermitian operator $I(t)$ which satisfies the equation

$$
\frac{d I}{d t}=\frac{\partial I}{\partial t}+\frac{1}{i \hbar}[I, H]=0
$$

In case that such invariant $I$ exists for a system and if it does not contain time derivative operators, the solutions of the Schrödinger equation (5) take the form

$$
\Psi(\vec{r}, t)=e^{i \alpha(t)} \Phi(\vec{r}, t),
$$

where $\Phi(\vec{r}, t)$ is an eigenfunction of $I(t)$ that entails a timeindependent eigenvalue and $\alpha(t)$ is a phase of the wave function that can be derived from

$$
\hbar \frac{d \alpha(t)}{d t}=\left\langle\Phi(\vec{r}, t)\left|\left(i \hbar \frac{\partial}{\partial t}-H\right)\right| \Phi(\vec{r}, t)\right\rangle .
$$


According to the Lewis-Riesenfeld method which is available for deriving quantum solutions, it is necessary to construct an invariant operator of the system. By solving (6), we have the following invariant operator [30]:

$$
I=U I_{0} U^{-1}
$$

where $U$ is a unitary operator of the form

$$
U=\exp \left(i \frac{M \dot{\rho}}{2 \hbar \rho} r^{2}\right),
$$

and $I_{0}$ is another invariant quantity that is given by

$$
I_{0}=\frac{1}{2}\left[\rho^{2}\left(p^{2}+\frac{\delta}{r^{2}}\right)+\frac{1}{\rho^{2}} r^{2}\right] \text {. }
$$

Here, $\rho$ is the solution of an auxiliary equation which is

$$
\ddot{\rho}+\frac{\dot{M}}{M} \dot{\rho}+\omega^{2} \rho=\frac{1}{M^{2} \rho^{3}},
$$

where $M=M_{0}(1+\varepsilon t)^{2}$ and $\omega=\omega_{0} e^{(k / 2) t}$.

Now, we try to solve (12). The transformation $\rho=(1 /(1+$ $\varepsilon t)) y$ leads to the Yermakov's differential equation [31] which is

$$
\ddot{y}+\omega^{2} y=\frac{1}{M_{0}^{2} y^{3}} .
$$

Through the aid of the solution of this equation, we obtain the solution for $\rho(t)$ to be

$$
\rho(t)=\frac{W(t)}{\sqrt{C_{1}}(1+\varepsilon t)}\left[\frac{1}{M_{0}^{2}}+\left(C_{2}+C_{1} Z(t)\right)^{2}\right]^{1 / 2},
$$

where $C_{1}$ and $C_{2}$ are arbitrary real constants, and $W(t)$ and $Z(t)$ are given by

$$
\begin{aligned}
& W(t)=\frac{1}{\left(2 \omega_{0} / K\right) e^{(K / 2) t}}\left[d_{1} \sin \left(\frac{2 \omega_{0}}{K} e^{(K / 2) t}\right)\right. \\
& \left.-d_{2} \cos \left(\frac{2 \omega_{0}}{K} e^{(K / 2) t}\right)\right], \\
& \quad(t) \\
& \quad \frac{2}{K}\left\{\frac{\left(2 \omega_{0} / K\right) e^{(K / 2) t} \sin \left(\left(2 \omega_{0} / K\right) e^{(K / 2) t}\right)}{d_{2}\left[d_{2} \cos \left(\left(2 \omega_{0} / K\right) e^{(K / 2) t}\right)-d_{1} \sin \left(\left(2 \omega_{0} / K\right) e^{(K / 2) t}\right)\right]}\right. \\
& +\frac{\ln \left[d_{2} \cos \left(\left(2 \omega_{0} / K\right) e^{(K / 2) t}\right)-d_{1} \sin \left(\left(2 \omega_{0} / K\right) e^{(K / 2) t}\right)\right]}{d_{1}^{2}+d_{2}^{2}} \\
& \left.+\frac{d_{1}}{d_{2}\left(d_{1}^{2}+d_{2}^{2}\right)} \frac{2 \omega_{0}}{K} e^{(K / 2) t}\right\},
\end{aligned}
$$

where $d_{1}$ and $d_{2}$ are arbitrary constants.

\section{Eigenvalues and Eigenfunctions of the Invariant}

Let us express the eigenvalue equation for the invariant operator as

$$
I \Phi(\vec{r}, t)=\lambda \Phi(\vec{r}, t)
$$

where $\lambda$ is a time-independent eigenvalue. Because the wave functions for the time-dependent Hamiltonian system are represented in terms of the eigenfunction of the invariant operator, we now solve this equation in order to derive the eigenfunction $\Phi(\vec{r}, t)$. Through the use of the unitary relation with $U$, (17) can be mapped, in terms of $I_{0}$ given in (11), into

$$
I_{0} \phi(\vec{r})=\lambda \phi(\vec{r})
$$

where $\phi(\vec{r})$ is the eigenfunction of $I_{0}$. Notice that the relation between $\phi(\vec{r})$ and $\Phi(\vec{r}, t)$ is given by

$$
\phi(\vec{r})=U^{-1} \Phi(\vec{r}, t) .
$$

Using (11), the eigenvalue equation given in (18) can be written as

$$
\left\{\frac{\partial^{2}}{\partial \eta^{2}}+2 \frac{\partial}{\partial \eta}+\frac{1}{\hbar^{2}}\left[2 \lambda-\frac{L^{2}+\delta}{\eta^{2}}-\eta^{2}\right]\right\} \phi=0,
$$

where $\eta=r / \rho, \varphi=\tan ^{-1}(y / x), \theta=\cos ^{-1}(z / r)$, and $x, y, z$ are the Cartesian coordinates.

By taking the function $\phi$ to be

$$
\phi(\eta, \theta, \varphi)=\frac{1}{\eta} f(\eta) g(\theta, \varphi),
$$

and substituting this into (20), the differential equations for the radial and angular parts are separated as

$$
\begin{aligned}
& \frac{d^{2} f}{d \eta^{2}}+\frac{1}{\hbar^{2}}\left(2 \lambda-\frac{\hbar^{2} l(l+1)+\delta}{\eta^{2}}-\eta^{2}\right) f(\eta)=0 \\
& \left(\frac{\partial^{2}}{\partial \theta^{2}}+\cot \theta \frac{\partial}{\partial \theta}+l(l+1)+\frac{1}{\sin ^{2} \theta} \frac{\partial^{2}}{\partial \varphi^{2}}\right) g(\theta, \varphi) \\
& \quad=0
\end{aligned}
$$

where $l$ is the orbital momentum number. The solution of (23) is just the spherical harmonics:

$$
g(\theta, \varphi)=Y_{l}^{m}(\theta, \varphi) .
$$

By taking the transformation $s=\eta^{2}$, the radial equation leads to the following differential equation:

$$
\begin{aligned}
f^{\prime \prime} & +\frac{1}{2 s} f^{\prime}+\frac{1}{4 s^{2}}\left[-\frac{s^{2}}{\hbar^{2}}+\frac{2 \lambda}{\hbar^{2}} s-l(l+1)-\frac{\delta}{\hbar^{2}}\right] f \\
& =0
\end{aligned}
$$

We use the NU method [32] in order to solve this equation in the next section.

\section{The Nikiforov-Uvarov Method}

The NU method was proposed to treat complicated secondorder differential equations in a convenient way by reducing it to a hypergeometric-type equation through an appropriate 
coordinate transformation $s=s(t)$. To illustrate the general theory of this, let us consider a differential equation which is

$$
F^{\prime \prime}(s)+\frac{\tilde{\tau}(s)}{\sigma(s)} F^{\prime}+\frac{\tilde{\sigma}(s)}{\sigma(s)} F(s)=0,
$$

where $\sigma(s)$ and $\widetilde{\sigma}(s)$ are polynomials that are allowed up to the second order of $s$ and $\tilde{\tau}(s)$ is a polynomial allowed up to the first order of $s$. If we take the factorization

$$
F(s)=G(s) y(s),
$$

where $G(s)$ is defined through a logarithmic derivative of the form

$$
\frac{G^{\prime}(s)}{G(s)}=\frac{\pi(s)}{\sigma(s)}
$$

then, according to [33], the function $\pi$ required for this method is given by

$$
\pi(s)=\frac{\sigma^{\prime}-\tilde{\tau}}{2} \pm \sqrt{\left(\frac{\sigma^{\prime}-\tilde{\tau}}{2}\right)^{2}-\tilde{\sigma}-k \sigma},
$$

where

$$
k=-\pi^{\prime}-n \tau^{\prime}-\frac{n(n-1)}{2} \sigma^{\prime \prime},
$$

while the other variable $y(s)$ is the hypergeometric-type function whose polynomial solutions are given by the Rodrigues relation:

$$
y_{n}(s)=\frac{B_{n}}{\rho(s)} \frac{d^{n}}{d s^{n}}\left[\sigma^{n}(s) \rho(s)\right],
$$

where $B_{n}$ are normalizing constants and the weight function $\rho(s)$ must satisfy the condition

$$
(\sigma \rho)^{\prime}=\tau \rho
$$

with

$$
\tau(s)=\tilde{\tau}(s)+2 \pi(s) .
$$

For the case of a general form of the Schrödinger equation, which is given by

$$
\left[\frac{d^{2}}{d s^{2}}+\frac{\alpha_{1}-\alpha_{2} s}{s\left(1-\alpha_{3} s\right)} \frac{d}{d s}+\frac{-\zeta_{1} s^{2}+\zeta_{2} s-\zeta_{3}}{s^{2}\left(1-\alpha_{3} s\right)^{2}}\right] F(s)=0
$$

the value $k$ given in (30) can be represented as [33]

$$
k_{1,2}=-\beta_{1} \mp 2 \sqrt{\beta_{2}}
$$

where

$$
\begin{aligned}
& \beta_{1}=\frac{1}{2}\left(1-\alpha_{1}\right)\left[\alpha_{2}-\alpha_{3}\left(1+\alpha_{1}\right)\right]-\zeta_{2}+2 \alpha_{3} \zeta_{3}, \\
& \beta_{2}=\frac{\alpha_{3}^{2}}{16}\left[\left(1-\alpha_{1}\right)^{2}+4 \zeta_{3}\right] \gamma,
\end{aligned}
$$

with

$$
\gamma=\left(1+\alpha_{1}-\frac{\alpha_{2}}{\alpha_{3}}\right)^{2}+4\left(\frac{\zeta_{1}}{\alpha_{3}^{2}}-\frac{\zeta_{2}}{\alpha_{3}}+\zeta_{3}\right) .
$$

Again on the basis of the evaluation of [33], the general form of solution for $k=k_{1}=-\beta_{1}-2 \sqrt{\beta_{2}}$ is given by

$$
F(s)=s^{\beta_{3}}\left(1-\alpha_{3} s\right)^{\beta_{4}} P_{n}^{\left(\beta_{5}, \sqrt{\gamma}\right)}\left(1-2 \alpha_{3} s\right) \text {, }
$$

where

$$
\begin{aligned}
& \beta_{3}=\frac{1}{2}\left[1-\alpha_{1}+\sqrt{\left(1-\alpha_{1}\right)^{2}+4 \zeta_{3}}\right], \\
& \beta_{4}=\frac{1}{2}\left(1+\alpha_{1}-\frac{\alpha_{2}}{\alpha_{3}}\right)+\frac{1}{2} \sqrt{\gamma}, \\
& \beta_{5}=\sqrt{\left(1-\alpha_{1}\right)^{2}+4 \zeta_{3}},
\end{aligned}
$$

while, for $k=k_{2}=-\beta_{1}+2 \sqrt{\beta_{2}}$, the general solution is expressed as

$$
F(s)=s^{\tilde{\beta}_{3}}\left(1-\alpha_{3} s\right)^{\beta_{4}} P_{n}^{\left(-\beta_{5}, \sqrt{\gamma}\right)}\left(1-2 \alpha_{3} s\right),
$$

where

$$
\widetilde{\beta}_{3}=\frac{1}{2}\left[1-\alpha_{1}-\sqrt{\left(1-\alpha_{1}\right)^{2}+4 \zeta_{3}}\right] .
$$

By comparing (34) with (25), we get

$$
\begin{aligned}
& \alpha_{1}=\frac{1}{2}, \\
& \alpha_{2}=\alpha_{3}=0, \\
& \zeta_{1}=\frac{1}{\hbar^{2}}, \\
& \zeta_{2}=\frac{2 \lambda}{\hbar^{2}}, \\
& \zeta_{3}=l(l+1)+\frac{\delta}{\hbar^{2}} .
\end{aligned}
$$

At this stage, we should determine which kind of wave solutions is physically acceptable among the two given in (38) and (40), respectively. Because the wave solutions not only should be finite at $r=0$ but also should converge to zero at $r=\infty$, we can confirm that (38) is the only physically suitable kind of wave solutions among them. Then, after a minor evaluation regarding this, the solutions of (25) are given in terms of generalized Laguerre polynomials [34] such that

$$
f(r)=\left(\frac{r}{\rho}\right)^{\mu+1 / 2} \exp \left(-\frac{r^{2}}{\hbar \rho^{2}}\right) L_{n}^{\mu}\left(\frac{2 r^{2}}{\hbar \rho^{2}}\right),
$$

where $\mu=2 \sqrt{l(l+1)+1 / 16+\delta / \hbar^{2}}$, and the eigenvalue $\lambda$ of the invariant is expressed in the form

$$
\lambda_{n}=\frac{\hbar}{2}(2 n+1+\mu) .
$$


The complete normalized eigenfunctions of the invariant $I$ can be constructed by taking account of normalizing (21) with the use of the following relation [35]:

$$
\int_{0}^{\infty} e^{-x} x^{\mu}\left[L_{n}^{\mu}(x)\right]^{2} d x=\frac{\Gamma(\mu+n+1)}{n !} .
$$

Then, by gathering (19), (24), and (43) and introducing an appropriate factor $1 / \rho^{3 / 2}$ into these functions for the purpose of ensuring the normalization condition, we obtain

$$
\begin{aligned}
\Phi_{n, l, m}(r, \theta, \varphi, t)= & A_{n} \frac{1}{\rho^{3 / 2}}\left(\frac{r}{\rho}\right)^{\mu-1 / 2} \\
& \cdot \exp \left[\left(\frac{i M \rho \dot{\rho}}{2}-1\right) \frac{r^{2}}{\hbar \rho^{2}}\right] \\
& \cdot L_{n}^{\mu}\left(\frac{2 r^{2}}{\hbar \rho^{2}}\right) Y_{l}^{m}(\theta, \varphi),
\end{aligned}
$$

where the normalization constants are given by

$$
A_{n}=\sqrt{\frac{2^{\mu+2} n !}{\hbar^{\mu+1} \Gamma(\mu+n+1)}} .
$$

Hence, the exact solutions of the original Schrödinger equation given in (5), which is associated with the central harmonic plus an inverse harmonic potential, can now be obtained in the form

$$
\begin{gathered}
\Psi(r, \theta, \varphi, t)=\sqrt{\frac{2^{\mu+2} n !}{\rho^{3} \hbar^{\mu+1} \Gamma(\mu+n+1)}} e^{i \alpha(t)}\left(\frac{r}{\rho}\right)^{\mu-1 / 2} \\
\cdot \exp \left[\left(\frac{i M \rho \dot{\rho}}{2}-1\right) \frac{r^{2}}{\hbar \rho^{2}}\right] L_{n}^{\mu}\left(\frac{2 r^{2}}{\hbar \rho^{2}}\right) Y_{l}^{m}(\theta, \varphi),
\end{gathered}
$$

where the global phases are given by

$$
\alpha(t)=-\frac{1}{2}(2 n+1+\mu) \int_{0}^{t} \frac{d t^{\prime}}{M\left(t^{\prime}\right) \rho^{2}\left(t^{\prime}\right)} .
$$

These wave functions are useful for investigating quantum properties of the system. For example, the uncertainty relations between a Cartesian coordinate $x$ and its conjugate momentum are obtained through the use of these wave functions, such that

$$
\Delta x \Delta p_{x}=\hbar\left[1+M_{0}^{2}(1+\varepsilon t)^{4} \rho^{2} \dot{\rho}^{2}\right]^{1 / 2}\left(n+\frac{1}{2}\right) \geq \frac{\hbar}{2} .
$$

Thus, the ground state uncertainty product under the timedependent situation is $(\hbar / 2)\left[1+M^{2}(t) \rho^{2} \dot{\rho}^{2}\right]^{1 / 2}$. If the timedependence of the Hamiltonian disappears, the wave functions reduce to the familiar ones known for the timeindependent harmonic oscillator perturbed by the inverse harmonic potential and the uncertainty relations become $\Delta x \Delta p_{x}=\hbar(n+1 / 2)$ as expected.

\section{Conclusion}

Quantum solutions of the central time-dependent harmonic plus an inverse harmonic potential system in three dimensions were investigated using several mathematical techniques. According to the theory of Lewis and Risenfeld, the wave functions of the system are represented in terms of the eigenfunctions of the invariant operator. An exact invariant is established on the basis of its fundamental definition via the use of its unitary relation with the unitary operator given in (10). The invariant operator is represented in terms of a time function $\rho(t)$, while its overall time derivative results in zero. The eigenvalue equation for $I(t)$ has been written in terms of a second-order differential equation. To derive the solutions of such equation, the unitary transformation approach and the NU method are used together. The NU method enabled us to reduce the second-order differential equation to the hypergeometric equation. From these procedures, we have obtained the eigenfunctions and they are represented in terms of the Laguerre polynomials. The eigenfunctions of the invariant operator derived in this way are necessary for representing the Schrödinger solutions, that is, the wave functions. The finally obtained wave functions are expressed in terms of these eigenfunctions and time-dependent phase factors, given in (49), that correspond to global phases.

The difference of this research from the previous one, performed by Ferkous et al. [30], is that we used the NU method in order to derive exact quantum solutions of the system whereas Ferkous et al. did not. Quantum wave functions that we derived in this work were represented in terms of spherical harmonics $Y_{l}^{m}(\theta, \varphi)$, while those of Ferkous et al. were represented in terms of the Jacobi polynomials. Our results for the wave functions given in (48) are useful for investigating diverse fundamental quantum properties of the system. For example, they can be used to derive fluctuations of canonical variables, uncertainty relations, energy eigenvalues, Wigner functions, and so on. For a particular case where the time-dependence of parameters disappears, the wave functions (48) reduce to the familiar ones that are well known in quantum mechanics of the stationary system.

\section{Competing Interests}

The authors declare that they have no competing interests.

\section{Acknowledgments}

This research was supported by the Algerian Ministry of Higher Education and Scientific Research (Grant no. CNEPRU/D01220120010) and was supported by the Basic Science Research Program of the year 2015 through the National Research Foundation of Korea (NRF) funded by the Ministry of Education (Grant no. NRF-2013R1A1A2062907).

\section{References}

[1] F. Calogero, "Solution of a three-body problem in one dimension," Journal of Mathematical Physics, vol. 10, no. 12, pp. 21912196, 1969. 
[2] F. Calogero, "Solution of the one-dimensional $N$-body problems with quadratic and/or inversely quadratic pair potentials," Journal of Mathematical Physics, vol. 12, no. 3, pp. 419-436, 1971.

[3] B. Sutherland, "Quantum many-body problem in one dimension: ground state," Journal of Mathematical Physics, vol. 12, no. 2, pp. 246-250, 1971.

[4] V. V. Dodonov, I. A. Malkin, and V. I. Man'ko, "Green function and excitation of a singular oscillator," Physics Letters A, vol. 39, no. 5, pp. 377-378, 1972.

[5] V. V. Dodonov, I. A. Malkin, and V. I. Man'ko, "Excitations of a nonstationary asymmetrical singular oscillator," Il Nuovo Cimento B, vol. 24, no. 1, pp. 46-56, 1974.

[6] M. A. Markov, Ed., Invariants and the Evolution of Nonstationary Quantum Systems, Nova Science, New York, NY, USA, 1989.

[7] J. R. Choi and B. H. Kweon, "Operator method for a nonconservative harmonic oscillator with and without singular perturbation," International Journal of Modern Physics B, vol. 16, no. 31, pp. 4733-4742, 2002.

[8] I. A. Pedrosa, G. P. Serra, and I. Guedes, "Wave functions of a time-dependent harmonic oscillator with and without a singular perturbation," Physical Review A, vol. 56, no. 5, pp. 4300-4303, 1997.

[9] V. V. Dodonov, V. I. Man'ko, and L. Rosa, "Quantum singular oscillator as a model of a two-ion trap: an amplification of transition probabilities due to small-time variations of the binding potential," Physical Review A, vol. 57, no. 4, pp. 28512858, 1998.

[10] D. A. Trifonov, "Exact solutions for the general nonstationary oscillator with a singular perturbation," Journal of Physics A: Mathematical and General, vol. 32, no. 19, pp. 3649-3661, 1999.

[11] J.-R. Choi, "Exact quantum state for the time-dependent repulsive oscillator with a driving force and with a singular perturbation," Physica Scripta, vol. 70, no. 5, pp. 271-275, 2004.

[12] C.-I. Um, K.-H. Yeon, and T. F. George, “The quantum damped harmonic oscillator," Physics Reports, vol. 362, no. 2-3, pp. 63192, 2002.

[13] J.-R. Choi, "Quantum states with continuous spectrum for a general time-dependent oscillator," Pramana-Journal of Physics, vol. 65, no. 2, pp. 165-176, 2005.

[14] J.-R. Choi, “Thermal state of general time-dependent harmonic oscillator with a singular perturbation," Physica Scripta, vol. 68, no. 1, pp. 36-40, 2003.

[15] J. R. Choi, "Exact wave functions of time-dependent Hamiltonian systems involving quadratic, inverse quadratic, and $(1 / \widehat{x}) \hat{p}+\widehat{p}(1 / \hat{x})$ terms," International Journal of Theoretical Physics, vol. 42, no. 4, pp. 853-861, 2003.

[16] S. Menouar and J. R. Choi, "A hybrid approach for quantizing complicated motion of a charged particle in time-varying magnetic field," Annals of Physics, vol. 353, pp. 307-316, 2015.

[17] G. Lu, W. Hai, and L. Cai, "Near coherent states of an electron in a time-dependent linear potential," Physics Letters A, vol. 357, no. 3, pp. 181-187, 2006.

[18] M. S. Child, Semiclassical Mechanics with Molecular Applications, Oxford University Press, Oxford, UK, 1991.

[19] O. Éboli, R. Jackiw, and S.-Y. Pi, "Quantum fields out of thermal equilibrium," Physical Review D, vol. 37, no. 12, pp. 3557-3581, 1988.

[20] J. Bauer, "Comment on 'Solution of the Schrödinger equation for the time-dependent linear potential', Physical Review A, vol. 65, no. 3, Article ID 036101, 2002.
[21] H. R. Lewis Jr., "Classical and quantum systems with timedependent harmonic-oscillator-type hamiltonians," Physical Review Letters, vol. 18, no. 13, pp. 510-512, 1967.

[22] H. R. Lewis Jr. and W. B. Riesenfeld, "An exact quantum theory of the time-dependent harmonic oscillator and of a charged particle in a time-dependent electromagnetic field," Journal of Mathematical Physics, vol. 10, no. 8, pp. 1458-1473, 1969.

[23] S. M. Chumakov, V. V. Dodonov, and V. I. Man'ko, "Correlation functions of the nonstationary quantum singular oscillator," Journal of Physics A: Mathematical and General, vol. 19, no. 16, pp. 3229-3239, 1986.

[24] C. Yuce, "Exact solution of the time-dependent harmonic plus an inverse harmonic potential with a time-dependent electromagnetic field," Annals of Physics, vol. 308, no. 2, pp. 599604, 2003.

[25] S. V. Bulanov and F. Pegoraro, "Stability of a mass accreting shell expanding in a plasma," Physical Review E, vol. 65, no. 6, Article ID 066405, 2002.

[26] D. Mkrtichian, S.-L. Kim, A. V. Kusakin et al., "A search for pulsating, mass-accreting components in algol-type eclipsing binaries," in Close Binaries in the 21st Century: New Opportunities and Challenges, pp. 167-169, Springer, Amsterdam, The Netherlands, 2006.

[27] W. Kunkel and R. Harrington, "Modeling the motion of an increasing mass system," The Physics Teacher, vol. 48, no. 4, pp. 243-245, 2010

[28] H. Dekker, "Classical and quantum mechanics of the damped harmonic oscillator," Physics Reports, vol. 80, no. 1, pp. 1-110, 1981.

[29] J. R. Ray, "Dissipation and quantum theory," Lettere al Nuovo Cimento, vol. 25, no. 2, pp. 47-50, 1979.

[30] N. Ferkous, A. Bounames, and M. Maamache, "Timedependent Schrödinger equation with non-central potentials," Physica Scripta, vol. 88, no. 3, Article ID 035001, 2013.

[31] V. F. Zaitsev and A. D. Polyanin, Handbook of Exact Solution for Ordinary Differential Equations, CRC Press, Boca Raton, Fla, USA, 2002.

[32] A. F. Nikiforov and V. B. Uvarov, Special Function of Mathematical Physics, Birkhäuser, Basel, Switzerland, 1988.

[33] C. Tezcan and R. Sever, "A general approach for the exact solution of the Schrödinger equation," International Journal of Theoretical Physics, vol. 48, no. 2, pp. 337-350, 2009.

[34] Z. X. Wang and D. R. Guo, Special Functions, World Scientific, River Edge, NJ, USA, 1989.

[35] I. S. Gradshteyn and I. M. Ryzhik, Table of Integrals, Series, and Products, Academic Press, San Diego, Calif, USA, 8th edition, 2015. 


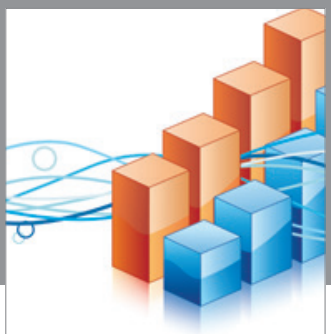

Advances in

Operations Research

vatem alat4

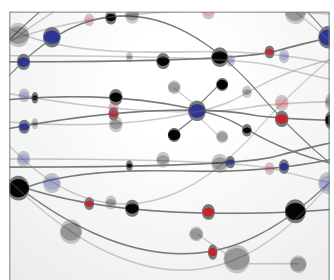

\section{The Scientific} World Journal
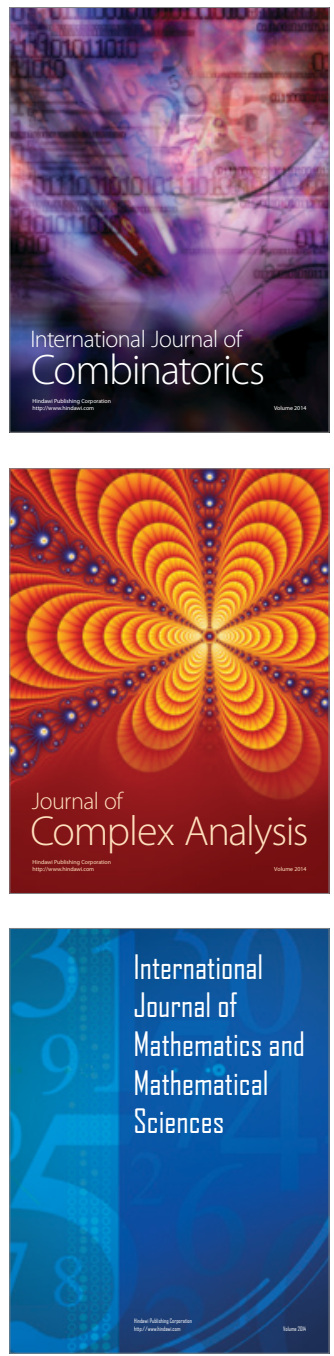
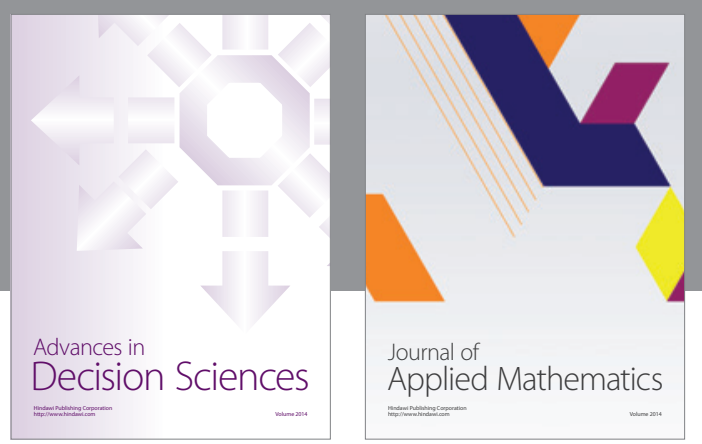

Algebra

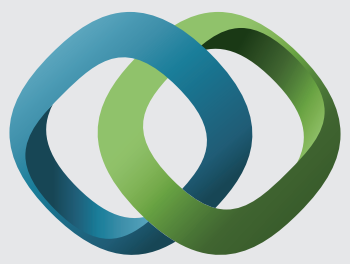

\section{Hindawi}

Submit your manuscripts at

http://www.hindawi.com
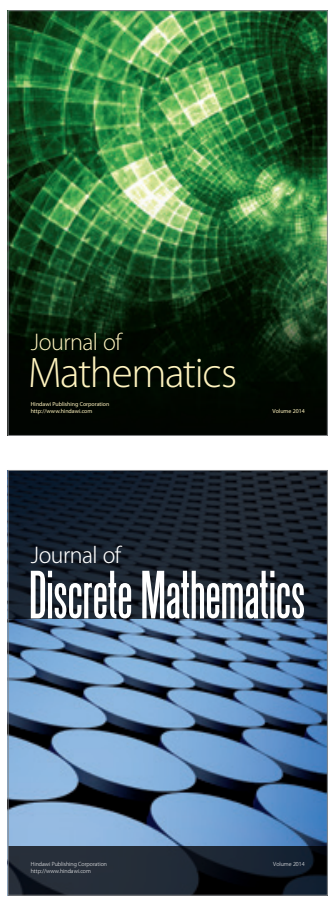

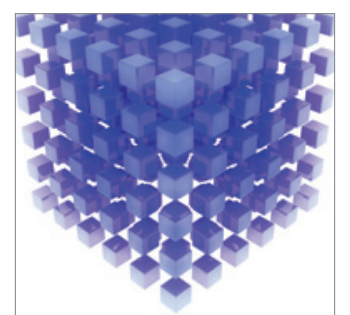

Mathematical Problems in Engineering
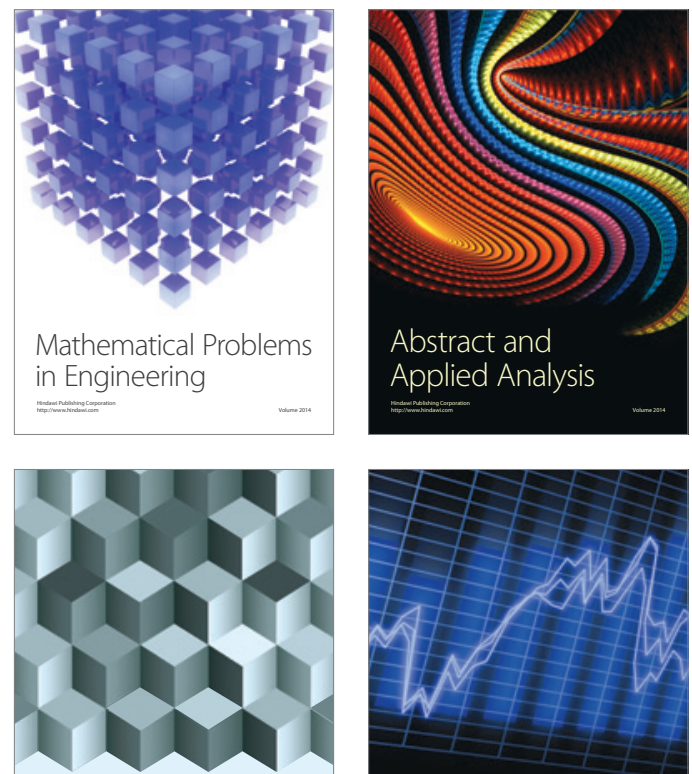

Journal of

Function Spaces

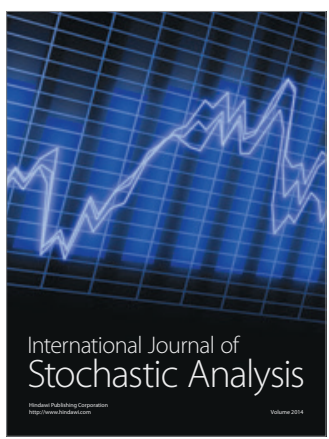

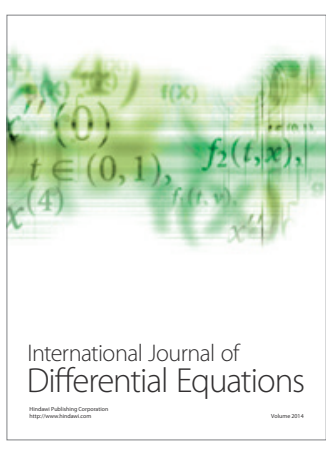
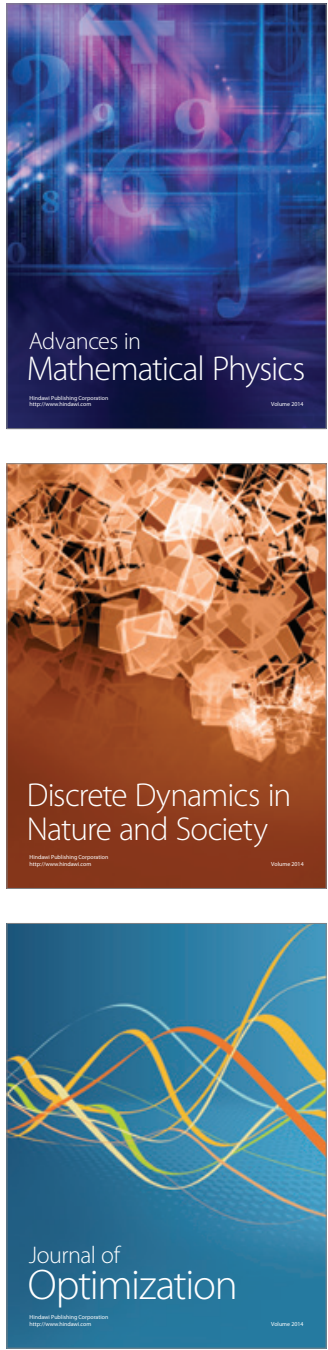